\title{
Challenging endeavor to integrate gallium and carbon via direct bonding to evolve GaN on diamond architecture
}

Jong Cheol Kim ${ }^{1,2}$, Jinhyung Lee ${ }^{6}$, Jongsik Kim ${ }^{5}$, Rajiv K. Singh ${ }^{2}$, Puneet Jawali ${ }^{2}$, Ghatu Subhash $^{4}$, Haigun Lee ${ }^{1, *}$ and Arul C. Arjunan ${ }^{3, *}$

${ }^{1}$ Department of Materials Science and Engineering

Korea University

145, Anam-dong, Seongbuk-gu, Seoul 02841, Republic of Korea

${ }^{2}$ Department of Materials Science and Engineering

University of Florida

Gainesville, FL 32611, United States

${ }^{3}$ Sinmat Inc.

1912 NW $67^{\text {th }}$ Place

Gainesville, FL 32653, United States

${ }^{4}$ Department of Mechanical and Aerospace Engineering

University of Florida

Gainesville, FL 32611, United States

${ }^{5}$ Korea Institute of Science and Technology

5, Hwarang-ro 14-gil, Seongbuk-gu, Seoul 02792, Republic of Korea

${ }^{6}$ SK Hynix

2091, Gyeongchung-daero, Bubal-eup, Icheon-si, Gyeonggi-do 17336, Republic of Korea

Keywords: GaN on diamond, spark plasma sintering, direct bonding, heat dissipation, immiscibility between $\mathrm{Ga}$ and $\mathrm{C}$

Abstract

*Corresponding author: haigunlee@korea.ac.kr; arul@ufl.edu 
This paper depicts efforts for fabricating GaN on diamond microstructure through direct bonding between $\mathrm{Ga}$ and $\mathrm{C}$, while excluding the use of adhesive interlayer during spark plasma sintering (SPS) process. The resulting GaN on diamond architecture is seemingly successful, as suggested by macroscopic morphological observations. The microscopic inspection using high-resolution transmission electron microscopy (HRTEM), however, shows a unique, off-the-chart interlayer configuration, wherein the components are migrated, etched, or fused to tentatively form multiple crystal phases. These phases can be constructed based on their utmost stabilities among all possible phases thermodynamically driven under or near the SPS conditions. 
Gallium nitride (GaN) on diamond structure is deemed to be of substantial interests in multiple electronic apparatus including high electron mobility transistor (HEMT), high power radio frequency (HPRF), light emitting diode (LED) devices, etc. [1-4]. This is because of such desired nature of GaN that provides wider band gap, higher thermal conductivity, and enhanced critical field strength/electron mobility compared to other Si-based competitors (e.g., Si and SiC) [5-8]. This, hence, allows for the operation of GaN-based devices at greater voltage with lower leakage current [9-11]. However, in spite of offering the beneficial properties as electronics, the GaNbased devices suffer from non-uniform heat distribution on an active GaN layer during the operation. This suppresses the efficient dissipation of heat from the active GaN layer, leading to exhibit low performance of the resulting GaN-based apparatus.

To avoid this limitation, recent GaN-based devices incorporate a heat spread layer that is directly attached to the device area. This layer plays a key role in distributing the heat across the entire spread layer to some extent (Figure 1a), yet, still opens a chance to further increase the efficiency associated with the heat elimination. It is suggested in the literatures that facilitated heat removal from the device can be realized by integrating materials with high thermal conductivity, among which diamond thin film is the most highly-profiled [12]. It should be noted that the production of active GaN layer on the thin diamond substrate is not an easy task and thus barely fabricated through the incorporation of an adhesion layer between GaN and diamond. Such example can be found on previous studies [13-16], wherein Cu heat sink is employed as an adhesion agent for the formation of the GaN on diamond architecture. In addition, recent works have also focused on developing a method denoted as top-down approach [17-19], which is typically initiated from the growth of the GaN thin film layer on a substrate such as SiC, Sapphire, or Si. 
Subsequently, a sacrificial wafer is immobilized on the growth side of the $\mathrm{GaN}$ thin film layer, whereas the substrate present in the opposite side of the GaN thin film layer is removed through grinding and/or polishing [17-19]. The resulting, ground GaN surface is then bound to a diamond substrate [17-19]. Of note, M. Kuball and co-workers recently produce GaN on diamond microstructure but still incorporate a dielectric interfacial layer for adhering the $\mathrm{GaN}$ to the diamond prior to removing the sacrificial wafer [19]. The utilization of the interfacial layer used in this binding strategy restricts the performance of the entire device because of the increase in the thermal resistance (Figure 1a) [19]. Alternatively, diamond thin film is suggested to be directly deposited onto GaN layers but turned out to evolve internal stresses at the interface. In particular, these stresses are detrimental to acquire stable and feasible performance of the device [20] and occasionally lead to the failure of the device due to the formation of micro-cracks [21]. Apparently, GaN on diamond microstructure via direct bonding with no adhesive agent has been rarely reported, to the best of our knowledge, and is our primary goal detailed and highlighted in this report.

For the creation of GaN-diamond architecture, we utilizes spark plasma sintering (SPS) process at $1000{ }^{\circ} \mathrm{C}$ with an uniaxial pressure of $40 \mathrm{MPa}$ for 2 minutes, as illustrated in Figure $1 b .^{\dagger}$ The resulting GaN-diamond microstructures are characterized using scanning electron microscope (SEM, JEOL-6400) for the observation of cross-sectional region. It is clarified in Figure $2 a$ that the distinct layers are evident, while showing feature of Si-GaN-diamond-Si architecture (i.e., different surface textures). Also, energy-dispersive X-ray spectroscopy (EDX) mapping was used to investigate the elemental composition of each layer. As shown in Figure 2b, 2c, and $2 \mathrm{~d}$, expected elements are found in corresponding interfacial 
regime, which can be another evidence to suggest the formation of Si-GaN-diamondSi structure.

High resolution transmission electron microscopy (HRTEM) is also employed to validate the production of GaN on diamond via chemical bonding between the outermost element in $\mathrm{GaN}$ (i.e., Ga) and diamond (i.e., C) during SPS process. Figure $3 a$ details the procedure to fabricate TEM specimen. ${ }^{\dagger}$ The Si substrate is first removed from the diamond region using a mechanical polishing and etching. Diamond thin film layer is then etched down to a thickness of c.a. $200 \mathrm{~nm}$ using inductively coupled plasma-reactive ion etching (ICP-RIE). After this, focused ion beam (FIB) system with SEM (FEI Strata DB235) is used to take the specimen comprising of polished GaN and diamond layers. Cross-sectional TEM (XTEM) is first examined on the interface layers. As shown in Figure $3 b$ and $3 c$, the interfacial regime seems eroded and exhibits abrupt shift in the relative position of layers when comparing to their locations originally designed (e.g., diamond $\leftrightarrow \mathrm{Si}$ ). To test whether FIB technique used for the specimens' preparation is responsible for their interfacial damage, we prepared additional TEM specimens following the identical method depicted above. However, interfacial disorder as well as off-the-chart layer configuration is consistent throughout all specimens analyzed in this work (Figure $4 a-4 d)$.

TEM analysis serves to establish a feasible mechanism accounting for the unexpected microstructure fabricated in this work. Given in literatures about SPS process potentially generating localized hot spots on the interface [22, 23], it is not surprising that the interfaces in microstructures are subjected to be physically deformed, chemically transformed into other crystal phases with physical phase transitions [22]. The physical deformation is evident in HRTEM images (Figure 3b 
and 3c), where interfaces include highly convective, diffused area with hollowed spots. In addition, GaN can be chemically decomposed in the presence of 'spark plasma' to yield Ga derivatives and high active $\mathrm{N}$ species. This is in close line with previous literature reports demonstrating that highly energetic plasma, which could overheat the specimens than that of operating temperature [22]. Based on that phenomena, it can alter bulk phase of $\mathrm{GaN}$ to form $\mathrm{Ga}$ and N, wherein the resulting liquid $\mathrm{Ga}$ (melting point of $30^{\circ} \mathrm{C}$ ) can corrode other species near the interface or migrate them through melting via energy transfer (i.e., Si in Figure $3 b$ and $3 c$ ). It is also highly likely that Si phase is modified by $\mathrm{N}$ or $\mathrm{C}$ (i.e., diamond) species to form bonding with Si-N or Si-C features, both of which are thermodynamically favorable under the given SPS condition [24]. In contrast, there is no solid solution reported between $\mathrm{Ga}$ and $\mathrm{Si}$, to date. This is because Ga-Si binary diagram shows a simple eutectic system with eutectic temperature close to c.a. $30{ }^{\circ} \mathrm{C}$ [25]. Additional hypothesis associated with Ga-C bonding is corroborated by Gibbs free energy calculation at $1000{ }^{\circ} \mathrm{C}$, as given in the following equations [26]. The positive Gibbs free energy $\left(\Delta \mathrm{G}^{\circ}{ }_{\mathrm{Ga} 4 \mathrm{C} 3}\right)$ indicates that the chemical fusion between $\mathrm{Ga}$ and $\mathrm{C}$ is unfavorable at the given temperature, which is mainly ascribed to large endothermic heat required to form $\mathrm{Ga}_{4} \mathrm{C}_{3}$ and therefore not accomplished in this work. Notably, the resulting GaN on diamond microstructure fabricated in this work can be stabilized only up to $\leq 1,100{ }^{\circ} \mathrm{C}$, while showing no critical structural collapse. This corroborates that the interfacial temperature between GaN and diamond layers is very likely $\leq 1,100{ }^{\circ} \mathrm{C}$, where $\Delta \mathrm{G}^{\circ}{ }_{\mathrm{Ga} 4 \mathrm{C} 3}$ still exhibits positive value. This supports our interpretation about unfavorable nature of $\mathrm{Ga}$ to bind with $\mathrm{C}$ under the SPS process condition employed in this work. 


$$
\begin{gathered}
G a(s)+C(s)=G a_{4} C_{3}(s) \\
\Delta G^{\circ}=\Delta H^{\circ}-T \Delta S^{\circ} \\
\Delta H_{G a_{4} C_{3}}^{\circ}=80,000\left(\mathrm{Jmol}^{-1}\right) \\
\Delta S_{G a_{4} C_{3}}^{\circ}=7.9\left(\mathrm{~J} \mathrm{~K}^{-1} \mathrm{~mol}^{-1}\right) \\
\Delta G_{G a_{4} C_{3}}^{\circ}=70,000\left(\mathrm{~J} \mathrm{~mol}^{-1}\right)
\end{gathered}
$$

To sum up, GaN on diamond architecture attempts to be fabricated via SPS process and is found particular hard due to localized, hot molten zones of interlayer present in the spark plasma environment. This can potentially result in the creation of the interesting architecture, in which Si species modified by $\mathrm{N}$ or $\mathrm{C}$ occupy the middle layer, while dissecting diamond and GaN layers. This suggests Si-based species can be a good candidate as an adhesive layer for GaN on diamond microstructure because thermal resistance of $\mathrm{Si}$ derivatives is lower than that of dielectric materials and thus expects to readily release heat from the device during the operation. The construction of novel, thin Si-dissecting GaN on diamond architecture is our on-going research.

This work was supported by National Science Foundation NSF SBIR Project (\# 646586) and Department of Energy SBIR Project (\# DE-SC000-6438 and \# DESC000-7740).

Experimental specifics can be found in a thesis (University of Florida at department of Materials Science and Engineering) by Dr. Kim, J. C., which is entitled as 'Processing and Characterization of Diamond Surfaces for Wafer Bonding Applications'. 

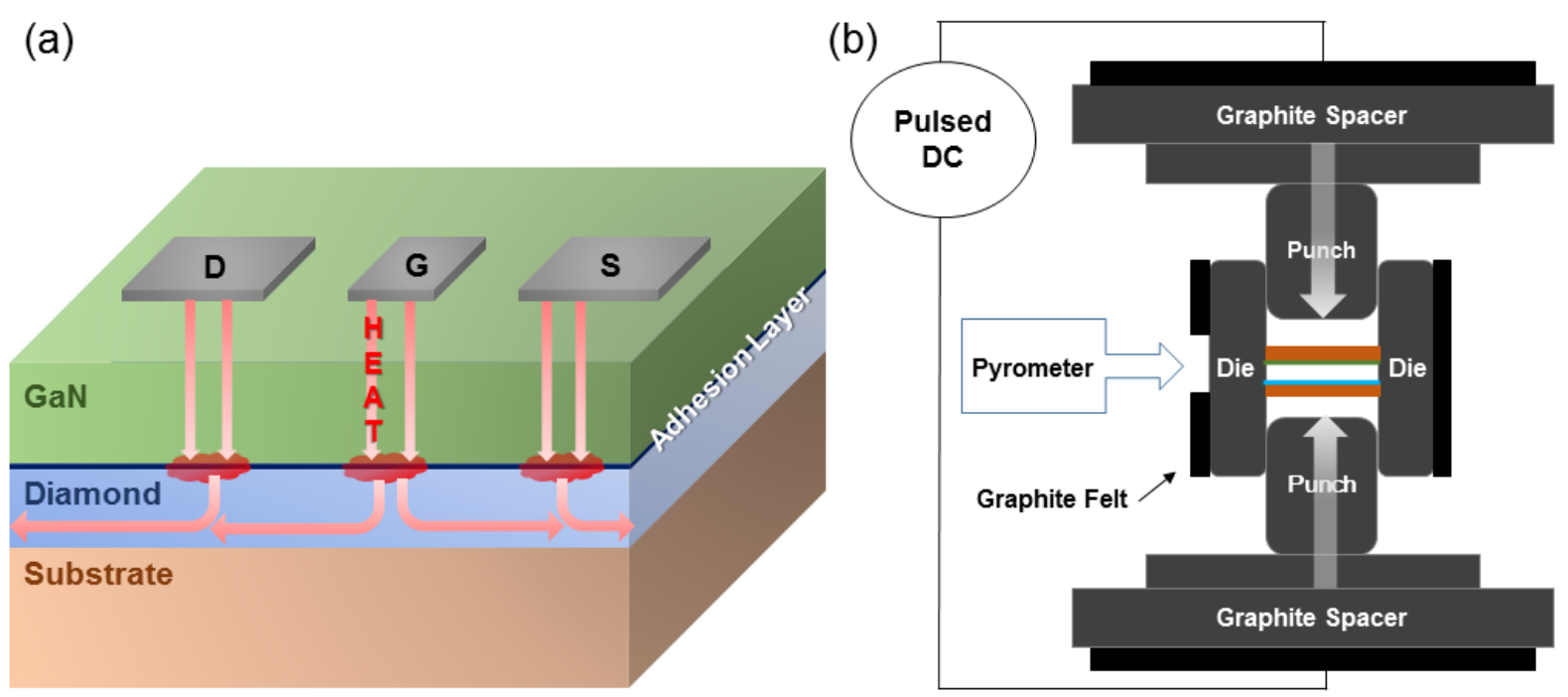

Figure 1. (a) Schematic representation of $\mathrm{GaN}$ on diamond architecture with the heat dissipation pathway shown as red arrows. D, G, and S denote drain, gate, and source, respectively. (b) Illustration of die assembly in spark plasma sintering (SPS) process. 

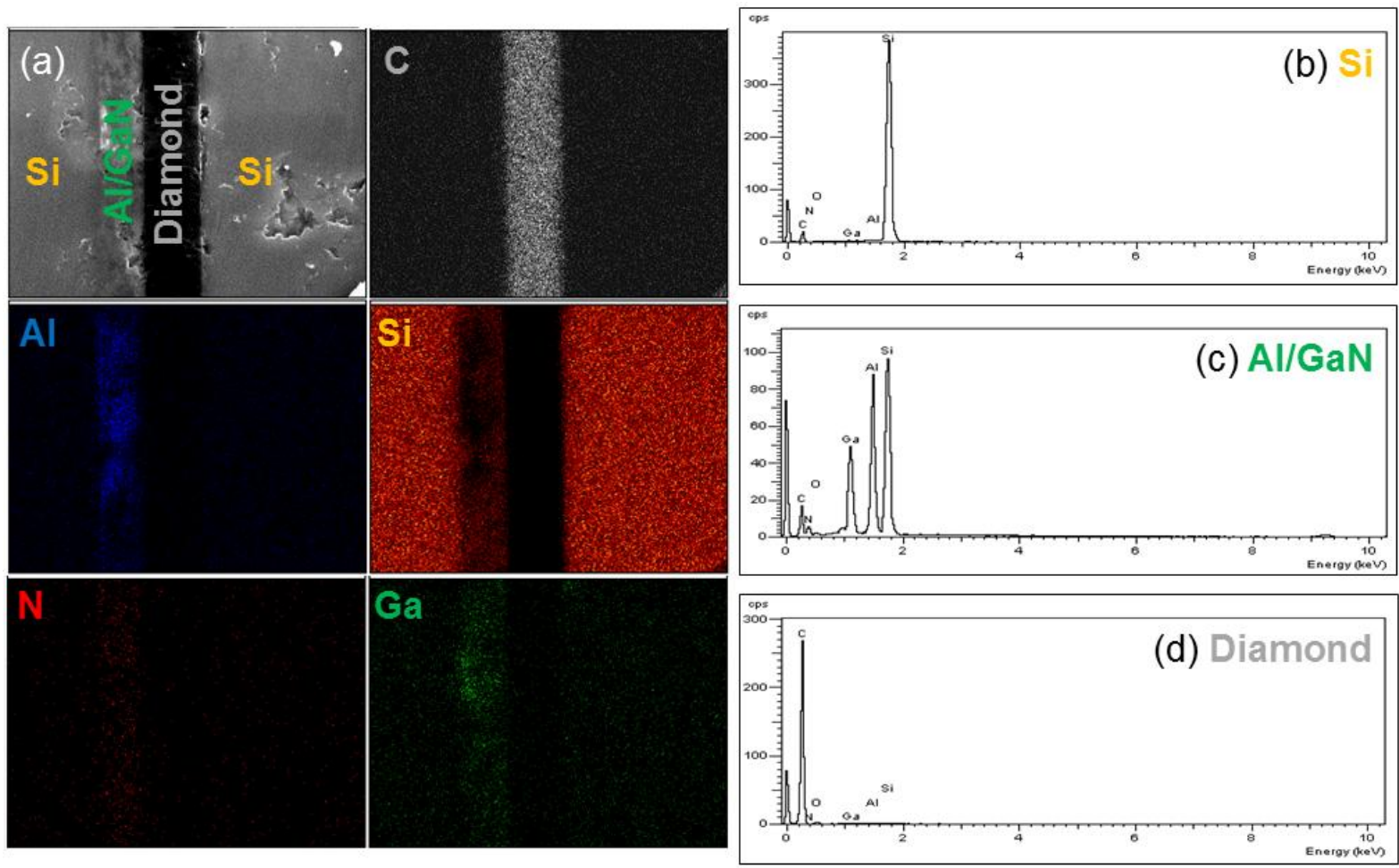

Figure 2. (a) Cross-sectional SEM image of GaN on diamond microstructure and EDS analysis of each layer (C: grey; Al: blue; Si: orange; N: red; Ga: green).EDS elemental mapping of $\mathrm{C}, \mathrm{Al}, \mathrm{Si}, \mathrm{N}$, and $\mathrm{Ga}$ at each layer: (b) $\mathrm{Si}$, (c) $\mathrm{Al} / \mathrm{GaN}$, and (d) Diamond. Al is also detected due to the presence of AlGaN buffer layer used for GaN growth on Si wafer. 


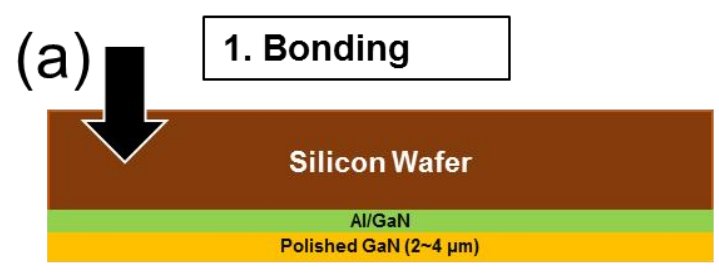

\section{Grinding \& Etching of Silicon Substrate}
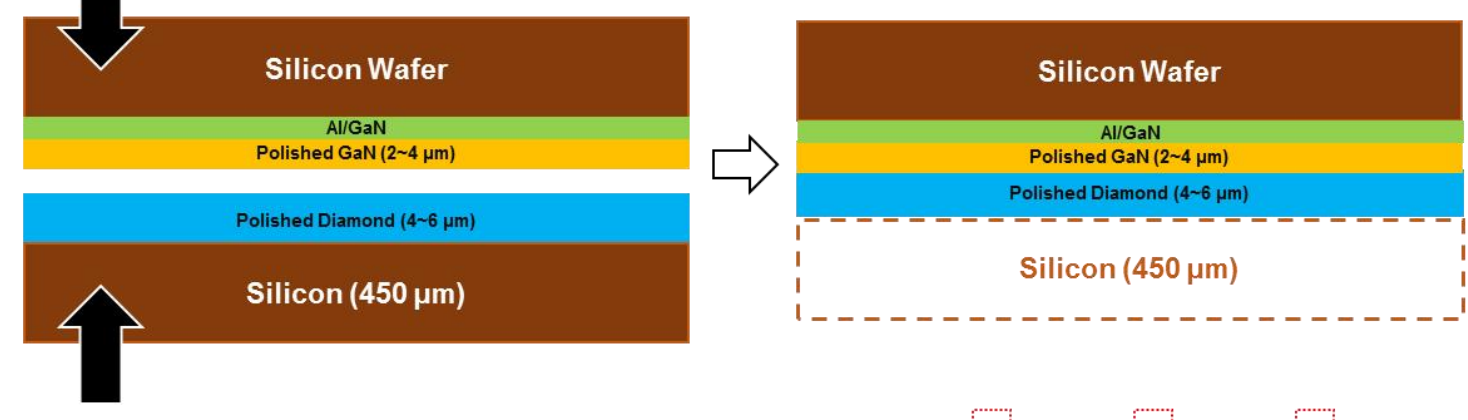

Polished Diamond ( 200 $\mathrm{nm})$

Polished GaN ( 200nm)

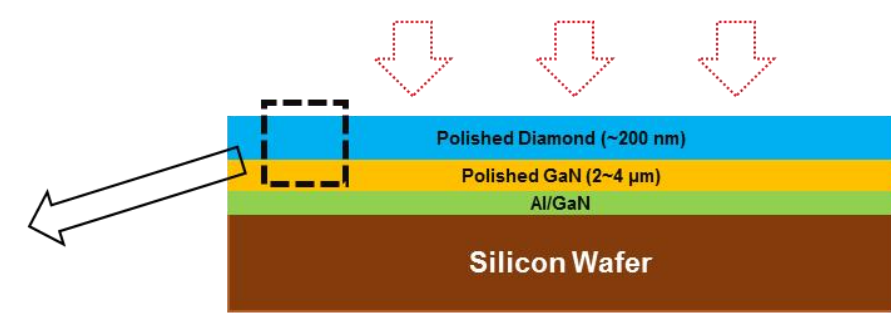

4. TEM Specimen by FIB

3. Diamond Thin Film Etching by RIE/ICP
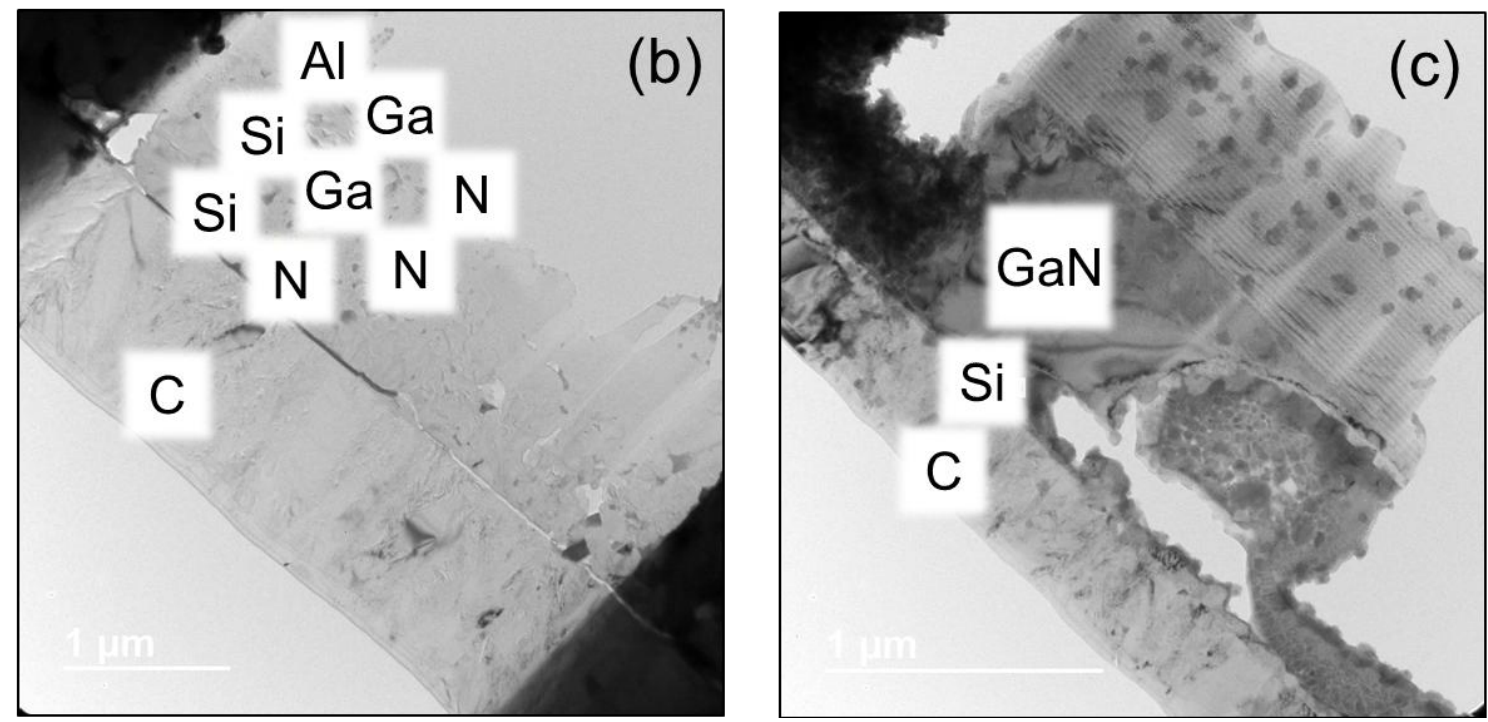

Figure 3. (a) Procedure to prepare FIB specimen preparation. (b-c) HRTEM images of GaN on diamond microstructure. 


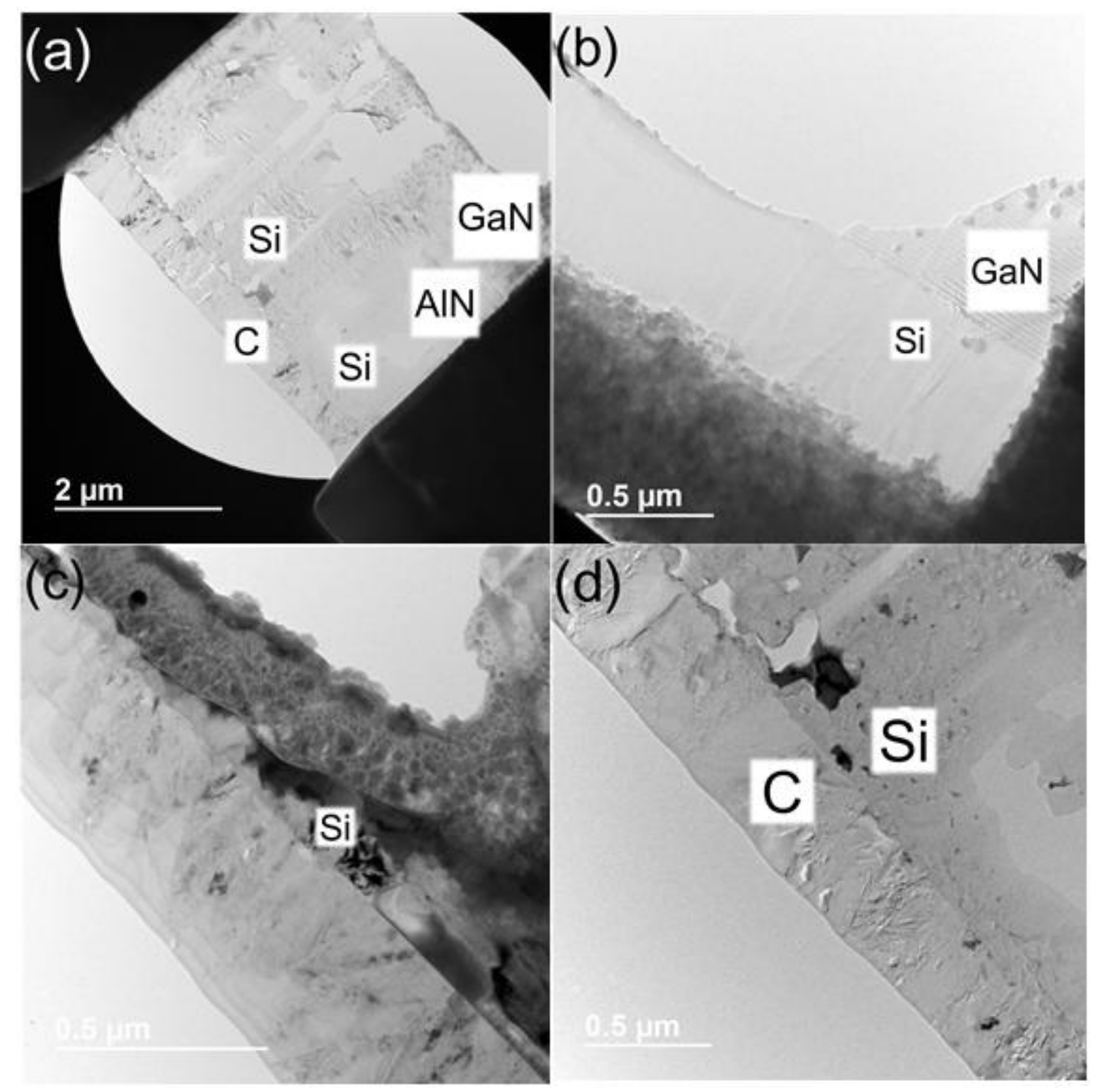

Figure 4. (a-d) HRTEM images of GaN on diamond specimens. 


\section{LIST OF REFERENCES}

[1] J.G. Felbinger, M. Chandra, Y. Sun, L.F. Eastman, J. Wasserbauer, F. Faili, D. Babic, D. Francis, F. Ejeckam, Electron Device Letters, IEEE 28(11) (2007) 948-950. [2] J.W. Zimmer, G. Chandler, Proc. CS MANTECH (2008) 14-17.

[3] B.J. Baliga, Semiconductor Science and Technology 28(7) (2013) 074011.

[4] R. Mitova, R. Ghosh, U. Mhaskar, D. Klikic, M.-X. Wang, A. Dentella, IEEE Transactions on Power Electronics 29(5) (2014) 2441-2452.

[5] P. Ye, B. Yang, K. Ng, J. Bude, G. Wilk, S. Halder, J. Hwang, Applied physics letters 86(6) (2005) 63501-63501.

[6] R. Bosshard, J.W. Kolar, IEEE Journal of Emerging and Selected Topics in Power Electronics 5(1) (2017) 419-431.

[7] G. Fiori, F. Bonaccorso, G. lannaccone, T. Palacios, D. Neumaier, A. Seabaugh, S.K. Banerjee, L. Colombo, Nature nanotechnology 9(10) (2014) 768-779.

[8] O. Hilt, E. Bahat-Treidel, A. Knauer, F. Brunner, R. Zhytnytska, J. Würfl, MRS Bulletin 40(5) (2015) 418-424.

[9] U.K. Mishra, P. Parikh, Y.-F. Wu, PROCEEDINGS-IEEE 90(6) (2002) 1022-1031. [10] Y. Yue, Z. Hu, J. Guo, B. Sensale-Rodriguez, G. Li, R. Wang, F. Faria, T. Fang, B. Song, X. Gao, Electron Device Letters, IEEE 33(7) (2012) 988-990.

[11] I.C. Kizilyalli, A.P. Edwards, O. Aktas, T. Prunty, D. Bour, IEEE Transactions on Electron Devices 62(2) (2015) 414-422.

[12] J. Cho, Z. Li, M. Asheghi, K.E. Goodson, Ann. Rev. Heat Transfer 18 (2014).

[13] D. Francis ${ }^{1}$, J. Wasserbauer ${ }^{1}$, F. Faili ${ }^{1}$, D. Babić ${ }^{1}$, F. Ejeckam ${ }^{1}$, W. Hong, P. Specht, E. Weber, (2007).

[14] F. Ejeckam, D. Francis, F. Faili, F. Lowe, D. Twitchen, B. Bolliger, GaN-onDiamond Wafers: Recent Developments, Semiconductor Technology International Conference (CSTIC), 2015 China, IEEE, 2015, pp. 1-3.

[15] Y. Han, B.L. Lau, X. Zhang, Y.C. Leong, K.F. Choo, Components, Packaging and Manufacturing Technology, IEEE Transactions on 4(6) (2014) 983-990.

[16] D.C. Dumka, P. Saunier, AIGaN/GaN HEMTs on diamond substrate, 2007 65th Annual Device Research Conference, 2007.

[17] D. Francis, F. Faili, D. Babić, F. Ejeckam, A. Nurmikko, H. Maris, Diamond and related materials 19(2-3) (2010) 229-233.

[18] V. Goyal, A.V. Sumant, D. Teweldebrhan, A.A. Balandin, Advanced Functional Materials 22(7) (2012) 1525-1530.

[19] D. Liu, D. Francis, F. Faili, C. Middleton, J. Anaya, J.W. Pomeroy, D.J. Twitchen, M. Kuball, Scripta Materialia 128 (2017) 57-60.

[20] S. Raghavan, J.M. Redwing, Journal of Applied Physics 98(2) (2005) 023514.

[21] D.J. Cheney, E.A. Douglas, L. Liu, C.-F. Lo, B.P. Gila, F. Ren, S.J. Pearton, Materials 5(12) (2012) 2498-2520.

[22] D. Giuntini, E.A. Olevsky, C. Garcia-Cardona, A.L. Maximenko, M.S. Yurlova, C.D. Haines, D.G. Martin, D. Kapoor, Materials 6(7) (2013) 2612-2632.

[23] W. Liu, M. Naka, Scripta Materialia 48(9) (2003) 1225-1230.

[24] D.R. Gaskell, Introduction to the Thermodynamics of Materials, CRC Press2008.

[25] R.W. Olesinski, N. Kanani, G. Abbaschian, Journal of Phase Equilibria 6(4) (1985) 362-364.

[26] B. Hájek, V. Kohout, V. Flemr, Monatshefte für Chemie / Chemical Monthly 117(10) (1986) 1157-1164. 
<GaN Thin Film Substrate on Silicon Wafer>

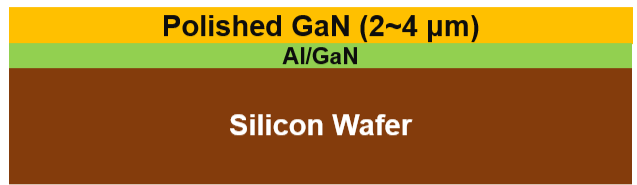

<Suggested GaN on Diamond Architecture>

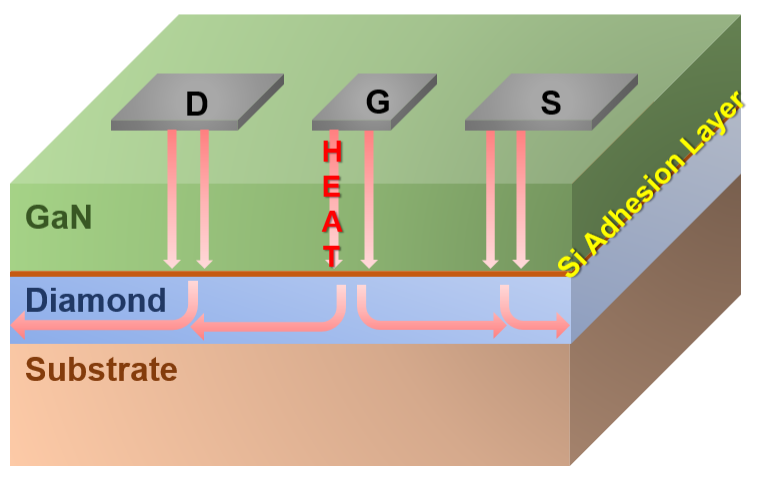

<Grinding \& Etching of Silicon Wafer>
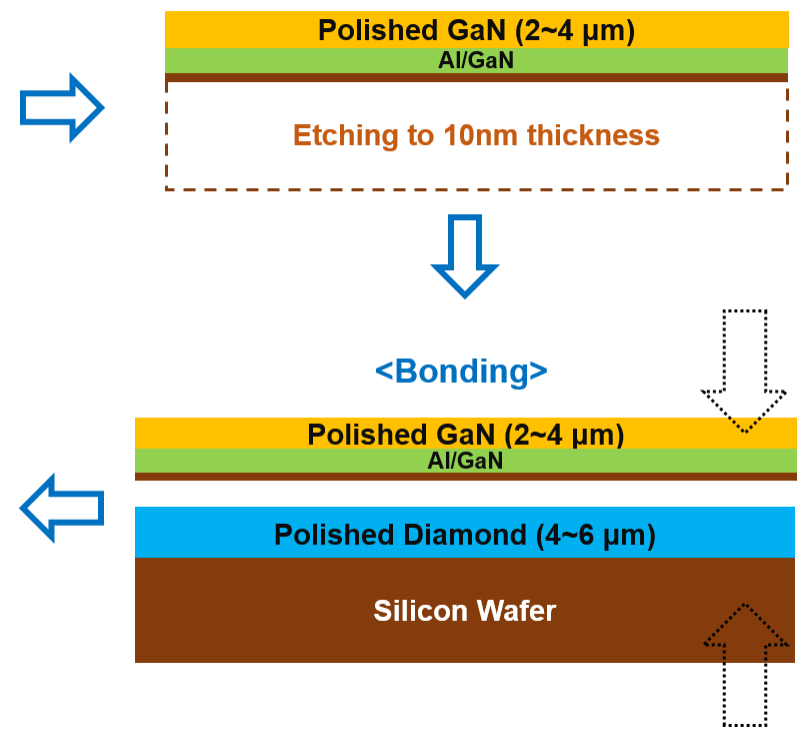